\title{
A new upper bound for finite additive bases
}

\author{
by \\ C. SinAn Güntürk (New York, NY) \\ and Melvyn B. Nathanson (Bronx, NY)
}

1. An extremal problem for finite bases. Let $\mathbb{N}_{0}$ and $\mathbb{Z}$ denote the nonnegative integers and integers, respectively, and let $|A|$ denote the cardinality of the set $A$.

Let $A$ be a set of integers, and consider the sumset

$$
2 A=\left\{a+a^{\prime}: a, a^{\prime} \in A\right\} .
$$

Let $S$ be a set of integers. The set $A$ is a basis of order 2 for $S$ if $S \subseteq 2 A$. The set $A$ is called a basis of order 2 for $n$ if the sumset $2 A$ contains the first $n$ nonnegative integers, that is, if $A$ is a basis of order 2 for the interval of integers $[0, n-1]:=\{0,1, \ldots, n-1\}$. We define $n(2, A)$ as the largest integer $n$ such that $A$ is a basis of order 2 for $n$, that is,

$$
n(2, A)=\max \{n:[0, n-1] \subseteq 2 A\} .
$$

Rohrbach [6] introduced the extremal problem of determining the largest integer $n$ for which there exists a set $A$ consisting of at most $k$ nonnegative integers such that $A$ is a basis of order 2 for $n$. Let

$$
n(2, k)=\max \left\{n(2, A): A \subseteq \mathbb{N}_{0} \text { and }|A|=k\right\} .
$$

Rohrbach's problem is to compute or estimate the extremal function $n(2, k)$. The set $A$ is called an extremal $k$-basis of order 2 if $|A| \leq k$ and $n(2, A)=$ $n(2, k)$.

For example, $n(2,1)=1$ and $n(2,2)=3$. The unique extremal 1-basis of order 2 is $\{0\}$, and the unique extremal 2-basis of order 2 is $\{0,1\}$. For $k=3$ we have $n(2,3)=5$, and the extremal 3 -bases of order 2 are $\{0,1,2\}$ and $\{0,1,3\}$. If $k \geq 2$ and $A$ is an extremal $k$-basis of order 2 , then $0,1 \in A$.

2000 Mathematics Subject Classification: Primary 11B13.

Key words and phrases: additive bases, segment bases, sumsets.

The work of S.G. was supported in part by NSF grant DMS 0219072. The work of M.B.N. was supported in part by grants from the NSA Mathematical Sciences Program and the PSC-CUNY Research Award Program. 
If $A$ is a finite set of $k$ nonnegative integers and $n(2, A)=n$, then $n \notin A$. If $a \in A$ and $a>n$, then the set $A^{\prime}=(A \backslash\{a\}) \cup\{n\}$ has cardinality $k$, and $n\left(2, A^{\prime}\right) \geq n+1>n(2, A)$. Therefore, if $A$ is an extremal $k$-basis of order 2 and $n(2, k)=n$, then

$$
\{0,1\} \subseteq A \subseteq\{0,1, \ldots, n-1\} \subseteq 2 A .
$$

If $A$ is an extremal $k$-basis for $n$, then $|A|=k$ and $A \subseteq\{0,1, \ldots, n-1\}$.

Rohrbach determined order of magnitude of $n(2, k)$. He observed that if $A$ is a set of cardinality $k$, then there are exactly $\left(\begin{array}{c}k+1 \\ 2\end{array}\right)$ ordered pairs of the form $\left(a, a^{\prime}\right)$ with $a, a^{\prime} \in A$ and $a \leq a^{\prime}$. This gives the upper bound

$$
n(2, k) \leq\left(\begin{array}{c}
k+1 \\
2
\end{array}\right)=\frac{k^{2}}{2}+O(k) .
$$

To derive a lower bound, he set $r=[k / 2]$ and constructed the set

$$
A=\{0,1, \ldots, r-1, r, 2 r, \ldots,(r-1) r\} .
$$

We have

$$
|A|=2 r-1 \leq k
$$

and $\left\{0,1, \ldots, r^{2}\right\} \subseteq 2 A$. Then

$$
n(2, A) \geq r^{2}+1 \geq(k-1)^{2} / 4+1=k^{2} / 4+O(k)
$$

and so

$$
n(2, k) \geq k^{2} / 4+O(k)
$$

Thus,

$$
\liminf _{n \rightarrow \infty} \frac{n(2, k)}{k^{2}} \geq \frac{1}{4}=0.25, \quad \limsup _{n \rightarrow \infty} \frac{n(2, k)}{k^{2}} \leq \frac{1}{2}=0.5 .
$$

It is an open problem to compute these upper and lower limits. Mrose [5] (see also [1]) proved that

$$
\liminf _{n \rightarrow \infty} \frac{n(2, k)}{k^{2}} \geq \frac{2}{7}=0.2857 \ldots,
$$

and this is still the best lower bound. Rohrbach used a combinatorial argument to get the nontrivial upper bound

$$
\limsup _{n \rightarrow \infty} \frac{n(2, k)}{k^{2}} \leq 0.4992 .
$$

Moser [3] introduced a Fourier series argument to obtain

$$
\limsup _{n \rightarrow \infty} \frac{n(2, k)}{k^{2}} \leq 0.4903,
$$

and subsequent improvements by Moser, Pounder, and Riddell [4] produced

$$
\limsup _{n \rightarrow \infty} \frac{n(2, k)}{k^{2}} \leq 0.4847 \text {. }
$$


Combining Moser's analytic method and Rohrbach's combinatorial technique, Klotz [2] proved that

$$
\limsup _{n \rightarrow \infty} \frac{n(2, k)}{k^{2}} \leq 0.4802 .
$$

In this paper, we use Fourier series for functions of two variables to obtain

$$
\limsup _{n \rightarrow \infty} \frac{n(2, k)}{k^{2}} \leq 0.4789 .
$$

We note that Rohrbach used a slightly different function $n(2, k)$ : He defined $n(2, k)$ as the largest integer $n$ for which there exists a set $A$ consisting of $k+1$ nonnegative integers such that the sumset $2 A$ contains the first $n+1$ nonnegative integers. Of course, Rohrbach's function and our function have the same asymptotics.

2. Moser's application of Fourier series. In this section we describe Moser's use of harmonic analysis to obtain an upper bound for $n(2, k)$. Let $A$ be an extremal $k$-basis of order 2 . Let $r_{2, A}(j)$ denote the number of representations of $j$ as a sum of two elements of $A$, that is,

$$
r_{2, A}(j)=\operatorname{card}\left(\left\{\left(a_{1}, a_{2}\right) \in A \times A: a_{1}+a_{2}=j \text { and } a_{1} \leq a_{2}\right\}\right) .
$$

We introduce the generating function

$$
f_{A}(q)=\sum_{a \in A} q^{a} .
$$

Then

$$
k=f_{A}(1)=|A| \quad \text { and } \quad \frac{f_{A}(q)^{2}+f_{A}\left(q^{2}\right)}{2}=\sum_{j \in 2 A} r_{2, A}(j) q^{j} .
$$

If $[0, n-1] \subseteq 2 A$, then $r_{2, A}(j) \geq 1$ for all $0 \leq j \leq n-1$. Hence there exist integers $\delta(j) \geq 0$ such that

$$
\frac{f_{A}(q)^{2}+f_{A}\left(q^{2}\right)}{2}=1+q+q^{2}+\cdots+q^{n-1}+\sum_{j \in 2 A} \delta(j) q^{j},
$$

where

$$
\delta(j)= \begin{cases}r_{2, A}(j)-1 & \text { if } j \in\{0,1, \ldots, n-1\} \\ r_{2, A}(j) & \text { otherwise }\end{cases}
$$

Let

$$
\Delta(q)=\sum_{j \in 2 A} \delta(j) q^{j}
$$

Then $\Delta(q) \geq 0$ for $q \geq 0$, and

$$
\frac{f_{A}(q)^{2}+f_{A}\left(q^{2}\right)}{2}=1+q+q^{2}+\cdots+q^{n-1}+\Delta(q) .
$$


Evaluating the generating function identity (1) at $q=1$, we obtain

$$
\left(k^{2}+k\right) / 2=n+\Delta(1) .
$$

Since $\Delta(1) \geq 0$, we have

$$
n \leq k^{2} / 2+O(k) \text {. }
$$

The strategy is to find a lower bound for $\Delta(1)$ of the form

$$
\Delta(1) \geq c k^{2}+O(k)
$$

for some $c>0$, and deduce

$$
n \leq\left(\frac{1}{2}-c\right) k^{2}+O(k)
$$

One obtains a simple combinatorial lower bound for $\Delta(1)$ by noting that if $a_{1}, a_{2} \in A$ and $n / 2 \leq a_{1} \leq a_{2}$, then $a_{1}+a_{2} \geq n$. Let $\ell$ denote the number of elements $a \in A$ such that $a \geq n / 2$. Then

$$
\Delta(1) \geq \sum_{j \geq n} \delta(j)=\sum_{j \geq n} r_{2, A}(j) \geq \frac{\ell(\ell+1)}{2} \geq \frac{\ell^{2}}{2} .
$$

Let $\omega=e^{2 \pi i / n}$ be a primitive $n$th root of unity. Let $r$ be an integer not divisible by $n$. Then

$$
1+\omega^{r}+\omega^{2 r}+\cdots+\omega^{(n-1) r}=0
$$

and so

$$
\frac{f_{A}\left(\omega^{r}\right)^{2}+f_{A}\left(\omega^{2 r}\right)}{2}=1+\omega^{r}+\omega^{2 r}+\cdots+\omega^{(n-1) r}+\sum_{j} \delta(j) \omega^{j r}=\Delta\left(\omega^{r}\right) .
$$

Applying the triangle inequality, we obtain

$$
\Delta(1) \geq\left|\Delta\left(\omega^{r}\right)\right|=\frac{\left|f_{A}\left(\omega^{r}\right)^{2}+f_{A}\left(\omega^{2 r}\right)\right|}{2} \geq \frac{\left|f_{A}\left(\omega^{r}\right)\right|^{2}-k}{2} .
$$

Let

$$
M=\max \left\{\left|f_{A}\left(\omega^{r}\right)\right|: r \not \equiv 0(\bmod n)\right\} .
$$

Then

$$
0 \leq M \leq k
$$

and

$$
\Delta(1) \geq\left(M^{2}-k\right) / 2 .
$$

It is also possible to obtain an analytic lower bound for $\Delta(1)$. For all integers $r$ not divisible by $n$, we have

$$
M \geq\left|f_{A}\left(\omega^{r}\right)\right|=\left|\sum_{a \in A} e^{2 \pi i r a / n}\right|=\left|\sum_{a \in A} \cos (2 \pi r a / n)+i \sin (2 \pi r a / n)\right|,
$$


and so

$$
\left|\sum_{a \in A} \cos (2 \pi r a / n)\right| \leq M, \quad\left|\sum_{a \in A} \sin (2 \pi r a / n)\right| \leq M .
$$

Let $\varphi(t)$ be a real-valued function with period 1 and with a Fourier series

$$
\varphi(t)=\sum_{r=0}^{\infty} a_{r} \cos (2 \pi r t)+\sum_{r=1}^{\infty} b_{r} \sin (2 \pi r t)
$$

whose Fourier coefficients are absolutely summable, that is,

$$
\sum_{r=0}^{\infty}\left|a_{r}\right|+\sum_{r=1}^{\infty}\left|b_{r}\right|<\infty
$$

Define $C=C(n)$ by

$$
C=\sum_{\substack{r=0 \\ n \mid r}}^{\infty}\left|a_{r}\right|
$$

For any integer $a$ we have

$$
\begin{aligned}
& \sum_{a \in A} \varphi\left(\frac{a}{n}\right)=\sum_{a \in A} \sum_{r=0}^{\infty} a_{r} \cos (2 \pi r a / n)+\sum_{a \in A} \sum_{r=1}^{\infty} b_{r} \sin (2 \pi r a / n) \\
& =\sum_{r=0}^{\infty} a_{r} \sum_{a \in A} \cos (2 \pi r a / n)+\sum_{r=1}^{\infty} b_{r} \sum_{a \in A} \sin (2 \pi r a / n) \\
& =\sum_{\substack{r=0 \\
n \nmid r}}^{\infty} a_{r} \sum_{a \in A} \cos (2 \pi r a / n)+\sum_{\substack{r=1 \\
n \nmid r}}^{\infty} b_{r} \sum_{a \in A} \sin (2 \pi r a / n)+k \sum_{\substack{r=0 \\
n \mid r}}^{\infty} a_{r},
\end{aligned}
$$

and so

$$
\left|\sum_{a \in A} \varphi\left(\frac{a}{n}\right)\right| \leq M \sum_{\substack{r=1 \\ n \nmid r}}^{\infty}\left(\left|a_{r}\right|+\left|b_{r}\right|\right)+k C .
$$

Let $\alpha_{1}$ and $\alpha_{2}$ be real numbers such that

$$
\varphi(t) \geq \begin{cases}\alpha_{1} & \text { for } 0 \leq t<1 / 2 \\ \alpha_{2} & \text { for } 1 / 2 \leq t<1\end{cases}
$$

Recall that $\ell$ denotes the number of elements $a \in A$ such that $n / 2 \leq a \leq$ $n-1$. Then

$$
\sum_{a \in A} \varphi\left(\frac{a}{n}\right) \geq(k-\ell) \alpha_{1}+\ell \alpha_{2}=k \alpha_{1}-\left(\alpha_{1}-\alpha_{2}\right) \ell
$$


We obtain the inequality

$$
k \alpha_{1}-\left(\alpha_{1}-\alpha_{2}\right) \ell \leq M \sum_{\substack{r=1 \\ n \nmid r}}^{\infty}\left(\left|a_{r}\right|+\left|b_{r}\right|\right)+k C .
$$

In this way, the function $\varphi(t)$ produces a lower bound for $M$, which, by (5), gives a lower bound for $\Delta(1)$.

Moser applied inequality (6) to the function

$$
\varphi(t)=\frac{1}{2} \cos (4 \pi t)+\sin (2 \pi t),
$$

whose nonzero Fourier coefficients are $a_{2}=1 / 2$ and $b_{1}=1$. Then $C=0$ for $n \geq 3$, and

$$
\left|\sum_{a \in A} \varphi\left(\frac{a}{n}\right)\right| \leq \frac{3 M}{2} .
$$

The function $\varphi(t)$ satisfies the inequality

$$
\varphi(t) \geq \begin{cases}1 / 2 & \text { for } 0 \leq t<1 / 2 \\ -3 / 2 & \text { for } 1 / 2 \leq t<1\end{cases}
$$

and so

$$
\sum_{a \in A} \varphi\left(\frac{a}{n}\right) \geq \frac{k-\ell}{2}-\frac{3 \ell}{2}=\frac{k-4 \ell}{2} .
$$

This implies that

$$
M \geq \frac{2}{3}\left|\sum_{a \in A} \varphi\left(\frac{a}{n}\right)\right| \geq \frac{k-4 \ell}{3} .
$$

Defining $\lambda=\ell / k$ and $\mu=M / k$, which both lie in $[0,1]$, we obtain the constraint

$$
\frac{4}{3} \lambda+\mu \geq \frac{1}{3}
$$

Recalling the combinatorial lower bound (3) and the analytical bound (5), we next obtain

$$
\frac{2 \Delta(1)}{k^{2}} \geq \max \left(\lambda^{2}, \mu^{2}-\frac{1}{k}\right) \geq \max (\lambda, \mu)^{2}-\frac{1}{k} .
$$

It is now easy to see that

$$
\max (\lambda, \mu) \geq \frac{\frac{4}{3} \lambda+\mu}{\frac{4}{3}+1} \geq \frac{1}{7},
$$

hence we obtain

$$
\Delta(1) \geq \frac{k^{2}}{98}-\frac{k}{2}
$$


Inserting this into inequality (2), we obtain

$$
\frac{k^{2}+k}{2}=n+\Delta(1) \geq n+\frac{k^{2}}{98}-\frac{k}{2},
$$

and so

$$
n \leq\left(\frac{1}{2}-\frac{1}{98}\right) k^{2}+k \leq 0.4898 k^{2}+k,
$$

which, in fact, has a slightly better constant than derived by Moser originally. The constant in this estimate can be further improved by optimizing the function $\varphi$.

In the next section we shall employ a more general method using Fourier series in two variables that ultimately yields an even better lower bound for $\Delta(1)$.

3. Fourier series in two variables. We use the same notation as in the previous section. In particular, $\ell$ denotes the number of integers $a \in A$ such that $a \geq n / 2$. Let $L$ denote the number of pairs $\left(a_{1}, a_{2}\right) \in A \times A$ such that $a_{1}+a_{2} \geq n$. Then $L \geq \ell^{2}$, and $k^{2}-L$ is the number of pairs $\left(a_{1}, a_{2}\right) \in A \times A$ such that $a_{1}+a_{2} \leq n-1$. We have the combinatorial lower bound

$$
\Delta(1) \geq \sum_{j \geq n} r_{2, A}(j)=(L+\ell) / 2 \geq L / 2 .
$$

Let $\varphi\left(t_{1}, t_{2}\right)$ be a real-valued function with period 1 in each variable and with a Fourier series

$$
\varphi\left(t_{1}, t_{2}\right)=\sum_{r_{1} \in \mathbb{Z}} \sum_{r_{2} \in \mathbb{Z}} \widehat{\varphi}\left(r_{1}, r_{2}\right) e^{2 \pi i r_{1} t_{1}} e^{2 \pi i r_{2} t_{2}}
$$

which converges absolutely, that is,

$$
\sum_{r_{1} \in \mathbb{Z}} \sum_{r_{2} \in \mathbb{Z}}\left|\widehat{\varphi}\left(r_{1}, r_{2}\right)\right|<\infty .
$$

We choose $\varphi\left(t_{1}, t_{2}\right)$ with zero mean, that is,

$$
\widehat{\varphi}(0,0)=\int_{0}^{1} \int_{0}^{1} \varphi\left(t_{1}, t_{2}\right) d t_{1} d t_{2}=0 .
$$

Let

$$
\begin{aligned}
& R_{1}=\left\{\left(t_{1}, t_{2}\right) \in[0,1) \times[0,1): t_{1}+t_{2}<1\right\}, \\
& R_{2}=\left\{\left(t_{1}, t_{2}\right) \in[0,1) \times[0,1): t_{1}+t_{2} \geq 1\right\} .
\end{aligned}
$$

If $a_{1}, a_{2} \in A$ and $a_{1}+a_{2} \leq n-1$, then $\left(a_{1} / n, a_{2} / n\right) \in R_{1}$. If $a_{1}+a_{2} \geq n$, then $\left(a_{1} / n, a_{2} / n\right) \in R_{2}$. 
Let $\alpha_{1}$ and $\alpha_{2}$ be real numbers such that

$$
\varphi\left(t_{1}, t_{2}\right) \geq \begin{cases}\alpha_{1} & \text { for }\left(t_{1}, t_{2}\right) \in R_{1} \\ \alpha_{2} & \text { for }\left(t_{1}, t_{2}\right) \in R_{2}\end{cases}
$$

We choose the function $\varphi\left(t_{1}, t_{2}\right)$ such that $\alpha_{1}>\alpha_{2}$. Then

$$
\sum_{a_{1} \in A} \sum_{a_{2} \in A} \varphi\left(\frac{a_{1}}{n}, \frac{a_{2}}{n}\right) \geq\left(k^{2}-L\right) \alpha_{1}+L \alpha_{2}=\alpha_{1} k^{2}-\left(\alpha_{1}-\alpha_{2}\right) L .
$$

We can rewrite this sum as follows:

$$
\begin{aligned}
\sum_{a_{1} \in A} \sum_{a_{2} \in A} \varphi\left(\frac{a_{1}}{n}, \frac{a_{2}}{n}\right) & =\sum_{a_{1} \in A} \sum_{a_{2} \in A} \sum_{r_{1} \in \mathbb{Z}} \sum_{r_{2} \in \mathbb{Z}} \widehat{\varphi}\left(r_{1}, r_{2}\right) e^{2 \pi i r_{1} a_{1} / n} e^{2 \pi i r_{2} a_{2} / n} \\
& =\sum_{r_{1} \in \mathbb{Z}} \sum_{r_{2} \in \mathbb{Z}} \widehat{\varphi}\left(r_{1}, r_{2}\right) \sum_{a_{1} \in A} e^{2 \pi i r_{1} a_{1} / n} \sum_{a_{2} \in A} e^{2 \pi i r_{2} a_{2} / n} \\
& =\sum_{r_{1} \in \mathbb{Z}} \sum_{r_{2} \in \mathbb{Z}} \widehat{\varphi}\left(r_{1}, r_{2}\right) f_{A}\left(\omega^{r_{1}}\right) f_{A}\left(\omega^{r_{2}}\right) .
\end{aligned}
$$

Consider the partition of the integer lattice $\mathbb{Z}^{2}=S_{0} \cup S_{1} \cup S_{2}$, where

$$
\begin{aligned}
S_{0}= & \left\{\left(r_{1}, r_{2}\right) \in \mathbb{Z}^{2}: r_{1} \equiv r_{2} \equiv 0(\bmod n)\right\}, \\
S_{1}= & \left\{\left(r_{1}, r_{2}\right) \in \mathbb{Z}^{2}: r_{1} \equiv 0(\bmod n), r_{2} \not \equiv 0(\bmod n)\right\} \\
& \cup\left\{\left(r_{1}, r_{2}\right) \in \mathbb{Z}^{2}: r_{1} \not \equiv 0(\bmod n), r_{2} \equiv 0(\bmod n)\right\}, \\
S_{2}= & \left\{\left(r_{1}, r_{2}\right) \in \mathbb{Z}^{2}: r_{1} \not \equiv 0(\bmod n), r_{2} \not \equiv 0(\bmod n)\right\} .
\end{aligned}
$$

We define $C_{i}=C_{i}(n)$ for $i=0,1,2$ by

$$
C_{i}=\sum_{\left(r_{1}, r_{2}\right) \in S_{i}}\left|\widehat{\varphi}\left(r_{1}, r_{2}\right)\right| .
$$

Recall that $\left|f_{A}\left(\omega^{r}\right)\right| \leq M$ if $r$ is not divisible by $n$ and $\left|f_{A}\left(\omega^{r}\right)\right|=k$ if $r$ is divisible by $n$. Then

$$
\left|\sum_{a_{1} \in A} \sum_{a_{2} \in A} \varphi\left(\frac{a_{1}}{n}, \frac{a_{2}}{n}\right)\right| \leq C_{0} k^{2}+C_{1} k M+C_{2} M^{2} .
$$

Combining (8) and (9), we obtain

$$
\alpha_{1} k^{2}-\left(\alpha_{1}-\alpha_{2}\right) L \leq C_{0} k^{2}+C_{1} k M+C_{2} M^{2} .
$$

Since $\alpha_{1}>\alpha_{2}$, we have

$$
L \geq \frac{\left(\alpha_{1}-C_{0}\right) k^{2}-C_{1} k M-C_{2} M^{2}}{\alpha_{1}-\alpha_{2}} .
$$

We again define $\mu=M / k$. Since $0 \leq M \leq k$, we have $0 \leq \mu \leq 1$. By (7), we have $2 \Delta(1) \geq L$, and so

$$
\frac{2 \Delta(1)}{k^{2}} \geq \frac{L}{k^{2}} \geq \frac{\left(\alpha_{1}-C_{0}\right)-C_{1} \mu-C_{2} \mu^{2}}{\alpha_{1}-\alpha_{2}} .
$$


By (5), we also have $2 \Delta(1) \geq M^{2}-k$, and so

$$
\frac{2 \Delta(1)}{k^{2}} \geq \max \left(\mu^{2}, \frac{\left(\alpha_{1}-C_{0}\right)-C_{1} \mu-C_{2} \mu^{2}}{\alpha_{1}-\alpha_{2}}\right)-\frac{1}{k} .
$$

Since the Fourier series of $\varphi\left(t_{1}, t_{2}\right)$ converges absolutely and since $\widehat{\varphi}(0,0)$ $=0$, we can arrange the Fourier series in the form of a sum over concentric squares

$$
\sum_{R=1}^{\infty} \sum_{\max \left(\left|r_{1}\right|,\left|r_{2}\right|\right)=R} \widehat{\varphi}\left(r_{1}, r_{2}\right) e^{2 \pi i r_{1} t_{1}} e^{2 \pi i r_{2} t_{2}} .
$$

For any $\varepsilon>0$ there exists an integer $N=N(\varepsilon)$ such that

$$
\sum_{n=N}^{\infty} \sum_{\max \left(\left|r_{1}\right|,\left|r_{2}\right|\right)=n}\left|\widehat{\varphi}\left(r_{1}, r_{2}\right)\right|<\varepsilon\left(\alpha_{1}-\alpha_{2}\right) .
$$

For all $n \geq N$, we shall approximate the sums $C_{0}, C_{1}$, and $C_{2}$ by $0, C_{\text {axial }}$, and $C_{\text {main }}$, respectively, where

$$
C_{\text {axial }}=\sum_{\substack{r \in \mathbb{Z} \\ r \neq 0}}(|\widehat{\varphi}(0, r)|+|\widehat{\varphi}(r, 0)|), \quad C_{\text {main }}=\sum_{\substack{r_{1} \in \mathbb{Z} \\ r_{1} \neq 0}} \sum_{\substack{r_{2} \in \mathbb{Z} \\ r_{2} \neq 0}}\left|\widehat{\varphi}\left(r_{1}, r_{2}\right)\right| .
$$

Then

$$
\begin{aligned}
\mid\left(\alpha_{1}-C_{0}\right)-C_{1} \mu-C_{2} \mu^{2}- & \left(\alpha_{1}-C_{\text {axial }} \mu-C_{\text {main }} \mu^{2}\right) \mid \\
& =\left|C_{0}+\left(C_{1}-C_{\text {axial }}\right) \mu+\left(C_{2}-C_{\text {main }}\right) \mu^{2}\right| \\
\leq & \left|C_{0}\right|+\left|C_{1}-C_{\text {axial }}\right|+\left|C_{2}-C_{\text {main }}\right| \\
\leq & \sum_{\max \left(\left|r_{1}\right|,\left|r_{2}\right|\right) \geq N}\left|\widehat{\varphi}\left(r_{1}, r_{2}\right)\right|<\varepsilon\left(\alpha_{1}-\alpha_{2}\right),
\end{aligned}
$$

and so

$$
\left|\frac{\left(\alpha_{1}-C_{0}\right)-C_{1} \mu-C_{2} \mu^{2}}{\alpha_{1}-\alpha_{2}}-\frac{\alpha_{1}-C_{\text {axial }} \mu-C_{\text {main }} \mu^{2}}{\alpha_{1}-\alpha_{2}}\right|<\varepsilon .
$$

It follows from inequality (10) that

$$
\frac{2 \Delta(1)}{k^{2}} \geq \max \left(\mu^{2}, \frac{\alpha_{1}-C_{\text {axial }} \mu-C_{\text {main }} \mu^{2}}{\alpha_{1}-\alpha_{2}}\right)-\varepsilon-\frac{1}{k} .
$$

Let

$$
\varrho=\inf _{0 \leq \mu \leq 1} \max \left(\mu^{2}, \frac{\alpha_{1}-C_{\text {axial }} \mu-C_{\text {main }} \mu^{2}}{\alpha_{1}-\alpha_{2}}\right) .
$$

From (10) and the definition of $\varrho$ in (11), we now have

$$
2 \Delta(1) / k^{2} \geq \varrho-\varepsilon-1 / k .
$$


Applying (2), we obtain

$$
\frac{k^{2}+k}{2}=n+\Delta(1) \geq n+\frac{(\varrho-\varepsilon) k^{2}-k}{2} .
$$

Therefore, we obtain the desired estimate

$$
n \leq \frac{1-\varrho+\varepsilon}{2} k^{2}+k
$$

for all sufficiently large $n$, where the number $\varrho$ depends only on the function $\varphi\left(t_{1}, t_{2}\right)$ and $\varepsilon>0$ can be arbitrarily small.

It is clear that we always have $\varrho \geq 0$, and that $\varrho>0$ if and only if $\alpha_{1}>0$. It is also clear that when $\alpha_{1} \geq 0$, we have $\varrho=\xi^{2}$, where $\xi$ is the unique solution in $[0,1]$ to the quadratic equation

$$
\xi^{2}=\frac{\alpha_{1}-C_{\text {axial }} \xi-C_{\text {main }} \xi^{2}}{\alpha_{1}-\alpha_{2}},
$$

i.e.,

$$
\left(\alpha_{1}-\alpha_{2}+C_{\text {main }}\right) \xi^{2}+C_{\text {axial }} \xi-\alpha_{1}=0,
$$

which yields the formula

$$
\varrho=\left(\frac{-C_{\text {axial }}+\sqrt{C_{\text {axial }}^{2}+4 \alpha_{1}\left(\alpha_{1}-\alpha_{2}+C_{\text {main }}\right)}}{2\left(\alpha_{1}-\alpha_{2}+C_{\text {main }}\right)}\right)^{2} .
$$

Hence we have an optimization problem in which we maximize $\varrho$ over all real-valued functions $\varphi$ defined on the unit square $[0,1)^{2}$ such that $\varphi$ has zero mean and $\varphi>0$ on $R_{1}$. We do not know the optimal function for this problem, but we have found a simple piecewise polynomial function that improves Klotz's upper bound for $n(2, k)$. Before we proceed to the main result of this paper, which also includes the definition of this function, let us present some of the heuristics which have lead us to our "educated guess."

First, without loss of generality, we may assume that $\alpha_{1}=1$. We then necessarily have

$$
0=\iint_{R_{1}} \varphi\left(t_{1}, t_{2}\right) d t_{1} d t_{2}+\iint_{R_{2}} \varphi\left(t_{1}, t_{2}\right) d t_{1} d t_{2} \geq \frac{1}{2}+\frac{1}{2} \alpha_{2},
$$

hence $\alpha_{2} \leq-1$. We also have

$$
C_{\text {axial }} \geq\left|\sum_{r}(\widehat{\varphi}(r, 0)+\widehat{\varphi}(0, r))\right|=\left|\int_{0}^{1}(\varphi(0, t)+\varphi(t, 0)) d t\right| \geq 2
$$

and

$$
C_{\text {main }} \geq\left|\sum_{r} \widehat{\varphi}(r, r)\right|=\left|\int_{0}^{1} \varphi(t, 1-t) d t\right| \geq 1 .
$$


In any case we are interested in the positive root $\xi_{\kappa, \tau}$ of the equation

$$
\kappa \xi^{2}+\tau \xi-1=0
$$

where $\kappa=1-\alpha_{2}+C_{\text {main }} \geq 3$ and $\tau=C_{\text {axial }} \geq 2$. Clearly, the smaller $\kappa$ and $\tau$ are, the larger this root will be. The bounds $\kappa \geq 3$ and $\tau \geq 2$ already imply that $\xi_{\kappa, \tau} \leq 1 / 3$, hence $\varrho=\xi_{\kappa, \tau}^{2} \leq 1 / 9$. In reality, $\alpha_{2}<-1$ because equality can happen only if $\varphi$ is constant on both $R_{1}$ and $R_{2}$, in which case $\widehat{\varphi}$ is not absolutely summable. This results in the heuristic that if we try to push $\alpha_{2}$ close to -1 , then $C_{\text {axial }}$ and $C_{\text {main }}$ will become large, and conversely if we try to push $C_{\text {axial }}$ and $C_{\text {main }}$ close to their respective minimum values, then $\varphi$ may not be bounded from below on $R_{2}$ by a small value. The right trade-off between these two competing quantities will result in the solution of this optimization problem.

It is interesting to note that the value of $\varrho$ is fairly robust with respect to variations in $\kappa$ and $\tau$, which we will only be able to estimate numerically and not compute exactly. The following lemma gives an explicit estimate for this purpose:

LEMMA 1. Let $\xi_{\kappa, \tau}$ and $\xi_{\kappa_{0}, \tau_{0}}$ be the respective positive roots of the equations $\kappa \xi^{2}+\tau \xi-1=0$ and $\kappa_{0} \xi^{2}+\tau_{0} \xi-1=0$. Let $\varrho=\xi_{\kappa, \tau}^{2}$ and $\varrho_{0}=\xi_{\kappa_{0}, \tau_{0}}^{2}$. If $\min \left(\kappa, \kappa_{0}\right) \geq 3$ and $\min \left(\tau, \tau_{0}\right) \geq 2$, then

$$
\left|\varrho-\varrho_{0}\right| \leq \frac{1}{54}\left|\kappa-\kappa_{0}\right|+\frac{1}{18}\left|\tau-\tau_{0}\right| .
$$

The proof of this lemma is given in the Appendix. Now we can state and prove the main theorem of this paper.

Theorem 1. We have

$$
\limsup _{n \rightarrow \infty} \frac{n(2, k)}{k^{2}} \leq 0.4789 .
$$

Proof. We define the function $\varphi\left(t_{1}, t_{2}\right)$ on the unit square $[0,1)^{2}$ by

$$
\begin{array}{r}
\varphi\left(t_{1}, t_{2}\right) \\
\quad= \begin{cases}1, & \left(t_{1}, t_{2}\right) \in R_{1}, \\
1-40\left(1-t_{1}\right)\left(1-t_{2}\right)\left(1-\left(2-t_{1}-t_{2}\right)^{6}\right), & \left(t_{1}, t_{2}\right) \in R_{2} .\end{cases}
\end{array}
$$

The graph of this function is plotted in Figure 1.

Clearly we have $\alpha_{1}=1$. Computation of the three other parameters used in formula (11) for $\varrho$ yields

$$
\begin{gathered}
\alpha_{2}=1-\frac{15}{2^{5 / 3}}=-3.72470 \ldots \\
2.90278 \leq C_{\text {axial }} \leq 2.90289, \quad 4.75145 \leq C_{\text {main }} \leq 4.76146 .
\end{gathered}
$$

Taking $\kappa_{0}=1+3.72471+4.76146=9.48617$ and $\tau_{0}=2.90289$, we obtain $\varrho_{0}>0.04240,\left|\kappa-\kappa_{0}\right|<0.01002$ and $\left|\tau-\tau_{0}\right|<0.00011$, so it follows that 


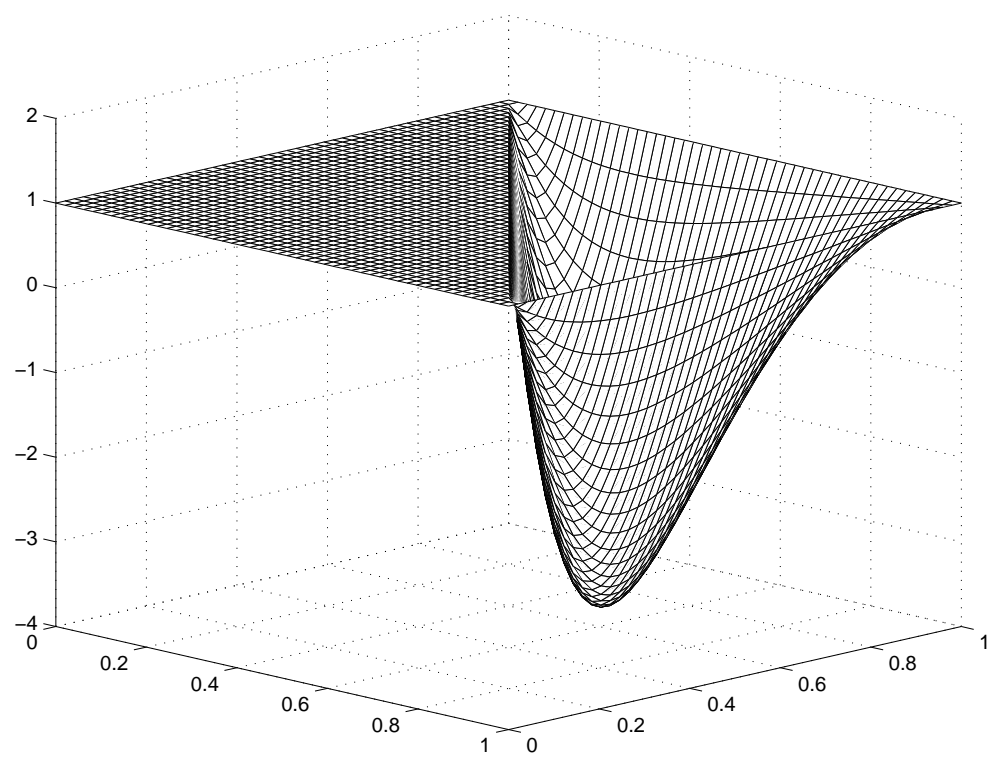

Fig. 1. Graph of the function $\varphi$ defined in (15)

$\varrho-\varrho_{0} \mid<0.0002$. Hence

$$
\varrho \geq \varrho_{0}-\left|\varrho-\varrho_{0}\right|>0.0422 .
$$

Consequently, choosing $\varepsilon$ sufficiently small and using (12), we obtain

$$
n \leq 0.4789 k^{2}+k \text {. }
$$

The details of the computations are in the Appendix to this paper. This completes the proof.

4. Open problems. A major open problem concerning the extremal function

$$
n(2, k)=\max \left\{n(2, A): A \subseteq \mathbb{N}_{0} \text { and }|A| \leq k\right\}
$$

is to compute $\liminf \operatorname{in}_{n \rightarrow \infty} n(2, k) / k^{2}$ and $\lim \sup _{n \rightarrow \infty} n(2, k) / k^{2}$, and to determine if the limit $\lim _{n \rightarrow \infty} n(2, k) / k^{2}$ exists. We have no conjecture about the existence of this limit, nor about the values of the lim inf and lim sup.

It is also difficult to compute the exact values of the function $n(2, k)$.

We can generalize the extremal functions $n(2, A)$ and $n(2, k)$ as follows. Let $A$ be a finite set of integers, and let $m(2, A)$ denote the largest integer $n$ such that the sumset $2 A$ contains $n$ consecutive integers. Let

$$
m(2, k)=\max \{m(2, A): A \subseteq \mathbb{Z} \text { and }|A| \leq k\} .
$$

Let $\ell(2, A)$ denote the largest integer $n$ such that the sumset $2 A$ contains 
an arithmetic progression of length $n$, and let

$$
\ell(2, k)=\max \{\ell(2, A): A \subseteq \mathbb{Z} \text { and }|A| \leq k\} .
$$

We can also define the extremal function

$$
n^{\prime}(2, k)=\max \{n(2, A): A \subseteq \mathbb{Z} \text { and }|A| \leq k\} .
$$

Then

$$
n(2, A) \leq n^{\prime}(2, A) \leq m(2, A) \leq \ell(2, A)
$$

and so

$$
n(2, k) \leq n^{\prime}(2, k) \leq m(2, k) \leq \ell(2, k) .
$$

For any integer $t$ and set $A$, we have the translation $A+t=\{a+t: a \in A\}$. The functions $\ell$ and $m$ are translation invariant, that is, $\ell(2, A+t)=\ell(2, A)$ and $m(2, A+t)=m(2, A)$. We also have the trivial upper bound $\ell(2, k) \leq$ $\left(\begin{array}{c}k+1 \\ 2\end{array}\right)$, but it is an open problem to obtain nontrivial upper bounds for any of the extremal functions $n^{\prime}(2, k), m(2, k)$, or $\ell(2, k)$.

Appendix. We describe here the computations.

Proof of Lemma 1. We start with the formula

$$
\xi_{\kappa, \tau}=\frac{-\tau+\sqrt{\tau^{2}+4 \kappa}}{2 \kappa}=\frac{2}{\tau+\sqrt{\tau^{2}+4 \kappa}} .
$$

We next evaluate the partial derivatives of $\xi_{\kappa, \tau}$ with respect to $\kappa$ and $\tau$ :

$$
\begin{aligned}
\frac{\partial \xi_{\kappa, \tau}}{\partial \kappa} & =-\frac{4}{\sqrt{\tau^{2}+4 \kappa}\left(\tau+\sqrt{\tau^{2}+4 \kappa}\right)^{2}}, \\
\frac{\partial \xi_{\kappa, \tau}}{\partial \tau} & =-\frac{2}{\sqrt{\tau^{2}+4 \kappa}\left(\tau+\sqrt{\tau^{2}+4 \kappa}\right)}
\end{aligned}
$$

from which it follows that in the set $\{(\kappa, \tau): \kappa \geq 3, \tau \geq 2\}$, we have

$$
\left|\frac{\partial \xi_{\kappa, \tau}}{\partial \kappa}\right| \leq \frac{1}{36} \quad \text { and } \quad\left|\frac{\partial \xi_{\kappa, \tau}}{\partial \tau}\right| \leq \frac{1}{12}
$$

These bounds then imply

$$
\left|\xi_{\kappa, \tau}-\xi_{\kappa_{0}, \tau_{0}}\right| \leq \frac{1}{36}\left|\kappa-\kappa_{0}\right|+\frac{1}{12}\left|\tau-\tau_{0}\right| .
$$

We then note that $\xi_{\kappa, \tau} \leq 1 / 3$, which yields

$$
\left|\varrho-\varrho_{0}\right|=\left|\xi_{\kappa, \tau}-\xi_{\kappa_{0}, \tau_{0}}\right|\left|\xi_{\kappa, \tau}+\xi_{\kappa_{0}, \tau_{0}}\right| \leq \frac{2}{3}\left|\xi_{\kappa, \tau}-\xi_{\kappa_{0}, \tau_{0}}\right|,
$$

hence the result of the lemma. 
Absolute summability of $\widehat{\varphi}$. While the result explained in this subsection is elementary, we will provide a certain amount of detail in its derivation because our main concern is more than absolute summability of $\widehat{\varphi}$. We would like to provide explicit estimates on the rate of the convergence; this will be necessary later in the section when we will analyze the accuracy of the numerical computation of the constants $C_{\text {main }}$ and $C_{\text {axial }}$.

LEMma 2. Let $f$ be a smooth function on $[0,1]$. Then for all $L \geq 0$ and $n \neq 0$, the following formula holds:

$$
\widehat{f}(n)=\sum_{k=0}^{L} \frac{f^{(k)}(0)-f^{(k)}(1)}{(2 \pi i n)^{k+1}}+\frac{\widehat{f^{(L+1)}}(n)}{(2 \pi i n)^{L+1}} .
$$

Proof. The case $L=0$ follows from integration by parts and the general case follows from iterating this result.

THEOREM 2. Let $F$ be a smooth function on $\mathbb{R}^{2}$ which vanishes on the boundary of $R_{2}$, i.e.,

$$
F(t, 1-t)=F(1, t)=F(t, 1)=0 \quad \text { for all } t \in[0,1] .
$$

Define

$$
\Psi_{F}\left(t_{1}, t_{2}\right)= \begin{cases}0 & \text { if }\left(t_{1}, t_{2}\right) \in R_{1} \\ F\left(t_{1}, t_{2}\right) & \text { if }\left(t_{1}, t_{2}\right) \in R_{2} .\end{cases}
$$

Then the Fourier series expansion of $\Psi_{F}$ is absolutely convergent.

Note. Later we will simply set $\varphi=\Psi_{F}+1$.

Proof. We will prove this result by deriving a suitable decay estimate on $\left|\widehat{\Psi}_{F}\left(r_{1}, r_{2}\right)\right|$, where

$$
\widehat{\Psi}_{F}\left(r_{1}, r_{2}\right)=\int_{0}^{1} e^{-2 \pi i r_{1} t_{1}}\left\{\int_{1-t_{1}}^{1} F\left(t_{1}, t_{2}\right) e^{-2 \pi i r_{2} t_{2}} d t_{2}\right\} d t_{1} .
$$

The case $r_{1}=0$ or $r_{2}=0$. Due to the symmetry on the assumptions on $F$, it suffices to consider only one of these cases. Let us assume that $r_{2}=0$. Define

$$
J_{1}\left(t_{1}\right)=\int_{1-t_{1}}^{1} F\left(t_{1}, t_{2}\right) d t_{2}
$$

so that

$$
\widehat{\Psi}_{F}\left(r_{1}, 0\right)=\widehat{J}_{1}\left(r_{1}\right) .
$$

Clearly we have $J_{1}(0)=J_{1}(1)=0$. Setting $L=1$ and $f=J_{1}$ in Lemma 2, we see that for $r_{1} \neq 0$,

$$
\left|\widehat{\Psi}_{F}\left(r_{1}, 0\right)\right| \leq \frac{1}{\left|2 \pi r_{1}\right|^{2}}\left(\left|J_{1}^{\prime}(0)-J_{1}^{\prime}(1)\right|+\int_{0}^{1}\left|J_{1}^{\prime \prime}\right|\right)=O\left(\frac{1}{r_{1}^{2}}\right) .
$$


With a similar estimate for $\left|\widehat{\Psi}_{F}\left(0, r_{2}\right)\right|$, we have

$$
\sum_{r \neq 0}\left(\left|\widehat{\Psi}_{F}(r, 0)\right|+\left|\widehat{\Psi}_{F}(0, r)\right|\right)<\infty
$$

The case $r_{1} \neq 0$ and $r_{2} \neq 0$. We will derive a general formula for $\widehat{\Psi}_{F}\left(r_{1}, r_{2}\right)$. To do this, we momentarily forget that $F$ vanishes on the boundary of $R_{2}$, and for $t \in[0,1]$, define the following functions:

$$
\begin{array}{llrl}
g_{0}(t)=F(t, 1-t), & h_{0}(t)=F(t, 1), \\
g_{1}(t)=\left(\partial_{2} F\right)(t, 1-t), & h_{1}(t)=\left(\partial_{2} F\right)(1, t), \\
g_{2}(t)=\left(\partial_{1} \partial_{2} F\right)(t, 1-t), & h_{2}(t)=\left(\partial_{1} \partial_{2} F\right)(t, 1), \\
g_{3}(t)=\left(\partial_{1} \partial_{2}^{2} F\right)(t, 1-t), & h_{3}(t)=\left(\partial_{1} \partial_{2}^{2} F\right)(1, t) .
\end{array}
$$

We start with the formula for $\widehat{\Psi}_{F}\left(r_{1}, r_{2}\right)$ above. Integrating by parts in the second variable, we obtain

$$
\begin{aligned}
\widehat{\Psi}_{F}\left(r_{1}, r_{2}\right) & =\int_{0}^{1} d t_{1} e^{-2 \pi i r_{1} t_{1}}\left\{\left[\frac{e^{2 \pi i r_{2} t_{2}}}{-2 \pi i r_{2}} F\left(t_{1}, t_{2}\right)\right]_{t_{2}=1-t_{1}}^{t_{2}=1}\right. \\
& \left.\quad-\int_{1-t_{1}}^{1} d t_{2} \frac{e^{-2 \pi i r_{2} t_{2}}}{-2 \pi i r_{2}}\left(\partial_{2} F\right)\left(t_{1}, t_{2}\right)\right\} \\
& =\frac{1}{(2 \pi i) r_{2}}\left(\widehat{g}_{0}\left(r_{1}-r_{2}\right)-\widehat{h}_{0}\left(r_{1}\right)+\widehat{\Psi}_{\partial_{2} F}\left(r_{1}, r_{2}\right)\right) .
\end{aligned}
$$

We apply the same method to $\widehat{\Psi}_{\partial_{2} F}\left(r_{1}, r_{2}\right)$, but integrate by parts in the first variable. This results in

$$
\begin{aligned}
\widehat{\Psi}_{F}\left(r_{1}, r_{2}\right)= & \frac{1}{(2 \pi i) r_{2}}\left(\widehat{g}_{0}\left(r_{1}-r_{2}\right)-\widehat{h}_{0}\left(r_{1}\right)\right) \\
& +\frac{1}{(2 \pi i)^{2} r_{1} r_{2}}\left(\widehat{g}_{1}\left(r_{1}-r_{2}\right)-\widehat{h}_{1}\left(r_{2}\right)+\widehat{\Psi}_{\partial_{1} \partial_{2} F}\left(r_{1}, r_{2}\right)\right) .
\end{aligned}
$$

We repeat the first two steps in the same order, which gives us

$$
\begin{aligned}
\widehat{\Psi}_{F}\left(r_{1}, r_{2}\right)= & \frac{1}{(2 \pi i) r_{2}}\left(\widehat{g}_{0}\left(r_{1}-r_{2}\right)-\widehat{h}_{0}\left(r_{1}\right)\right) \\
& +\frac{1}{(2 \pi i)^{2} r_{1} r_{2}}\left(\widehat{g}_{1}\left(r_{1}-r_{2}\right)-\widehat{h}_{1}\left(r_{2}\right)\right) \\
& +\frac{1}{(2 \pi i)^{3} r_{1} r_{2}^{2}}\left(\widehat{g}_{2}\left(r_{1}-r_{2}\right)-\widehat{h}_{2}\left(r_{1}\right)\right) \\
& +\frac{1}{(2 \pi i)^{4} r_{1}^{2} r_{2}^{2}}\left(\widehat{g}_{3}\left(r_{1}-r_{2}\right)-\widehat{h}_{3}\left(r_{2}\right)+\widehat{\Psi}_{\partial_{1}^{2} \partial_{2}^{2} F}\left(r_{1}, r_{2}\right)\right) .
\end{aligned}
$$

Note that from our assumptions on $F$, we have $g_{0}=h_{0}=h_{1}=0$. We will have two subcases: 
(1) $r_{1}=r_{2}=r$. In this case, we easily see from the second formula above that

$$
\left|\widehat{\Psi}_{F}(r, r)\right| \leq \frac{\left|\widehat{g}_{1}(0)\right|+\left\|\Psi_{\partial_{1} \partial_{2} F}\right\|_{1}}{4 \pi^{2} r^{2}}
$$

(2) $r_{1} \neq r_{2}$. This case is slightly more subtle. We first note that $g_{1}(1)=$ $h_{1}(0)=0$. It is also true that $g_{1}(0)=\left(\partial_{2} F\right)(0,1)$. To see this, note that $\left(\partial_{1} F\right)(0,1)=0$ and $\nabla F(0,1) \cdot(1,-1)=0$, both of which follow from the fact that $F$ vanishes on the boundary of $R_{2}$. The function $g_{1}$ being smooth otherwise, we conclude that

$$
\left|\widehat{g}_{1}\left(r_{1}-r_{2}\right)\right| \leq \frac{\left|g_{1}^{\prime}(1)-g_{1}^{\prime}(0)\right|+\left\|g_{1}^{\prime \prime}\right\|_{1}}{4 \pi^{2}\left|r_{1}-r_{2}\right|^{2}} .
$$

The estimates for $g_{2}$ and $h_{2}$ are simpler in nature. We use the bounds

$$
\begin{gathered}
\left|\widehat{g}_{2}\left(r_{1}-r_{2}\right)\right| \leq \frac{\left|g_{2}(1)-g_{2}(0)\right|+\left\|g_{2}^{\prime}\right\|_{1}}{2 \pi\left|r_{1}-r_{2}\right|}, \\
\left|\widehat{h}_{2}\left(r_{1}\right)\right| \leq \frac{\left|h_{2}(1)-h_{2}(0)\right|+\left\|h_{2}^{\prime}\right\|_{1}}{2 \pi\left|r_{1}\right|},
\end{gathered}
$$

as well as

$$
\left|\widehat{g}_{3}\left(r_{1}-r_{2}\right)\right| \leq\left\|g_{3}\right\|_{1}, \quad\left|\widehat{h}_{3}\left(r_{1}\right)\right| \leq\left\|h_{3}\right\|_{1}, \quad\left|\widehat{\Psi}_{\partial_{1}^{2} \partial_{2}^{2} F}\left(r_{1}, r_{2}\right)\right| \leq\left\|\Psi_{\partial_{1}^{2} \partial_{2}^{2} F}\right\|_{1} .
$$

Putting all these together, we see that

$$
\left|\widehat{\Psi}_{F}\left(r_{1}, r_{2}\right)\right|=O\left(\frac{1}{\left|r_{1} r_{2}\right|\left(r_{1}-r_{2}\right)^{2}}+\frac{1}{\left|r_{1}\left(r_{1}-r_{2}\right)\right| r_{2}^{2}}+\frac{1}{r_{1}^{2} r_{2}^{2}}\right),
$$

which is easily verified to be summable over all admissible values of $r_{1}$ and $r_{2}$. We will return to this shortly for a more explicit estimate.

Explicit numerical estimates. In this subsection we will work with the specific function $\varphi$ in (15) for which

$$
F\left(t_{1}, t_{2}\right)=-40\left(1-t_{1}\right)\left(1-t_{2}\right)\left(1-\left(2-t_{1}-t_{2}\right)^{6}\right) .
$$

We will numerically estimate $C_{\text {axial }}$ and $C_{\text {main }}$ using appropriate tail bounds on their defining infinite series. This procedure is illustrated in Figure 2.

Estimating the value of $C_{\text {axial }}$. Since $F$ is symmetric, we have $\widehat{\varphi}(r, 0)=$ $\widehat{\varphi}(0, r)$. We use the formula (19) to evaluate $\widehat{\varphi}\left(r_{1}, 0\right)$. It is a simple calculation to show that

$$
J_{1}\left(t_{1}\right)=-15\left(1-t_{1}\right)+\frac{240}{7}\left(1-t_{1}\right)^{2}-20\left(1-t_{1}\right)^{3}+\frac{5}{7}\left(1-t_{1}\right)^{9} .
$$

Using this expression, we find that $\left|J_{1}^{\prime}(0)-J_{1}^{\prime}(1)\right|=15$ and

$$
\int_{0}^{1}\left|J_{1}^{\prime \prime}\right| \leq\left(\int_{0}^{1}\left(J_{1}^{\prime \prime}\right)^{2}\right)^{1 / 2}=8 \sqrt{15} .
$$




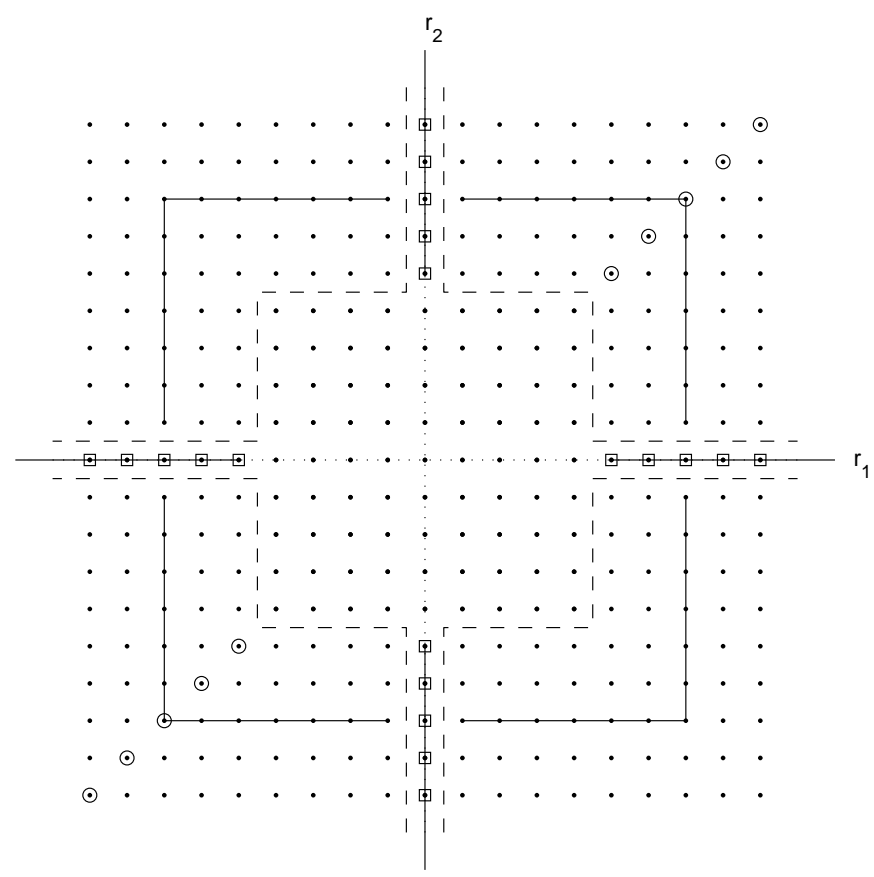

Fig. 2. Estimating the values of $C_{\text {axial }}$ and $C_{\text {main }}$. Circled dots correspond to the Fourier coefficients along the diagonal for which a separate decay estimate holds.

Hence by (20), we obtain the estimate

$$
|\widehat{\varphi}(r, 0)|=|\widehat{\varphi}(0, r)| \leq \frac{15+8 \sqrt{15}}{4 \pi^{2}} \frac{1}{r^{2}} .
$$

If we define

$$
C_{\text {axial }}(N)=\sum_{|r| \leq N}(|\widehat{\varphi}(r, 0)|+|\widehat{\varphi}(0, r)|),
$$

then it follows that

$$
0 \leq C_{\text {axial }}-C_{\text {axial }}(N) \leq \frac{15+8 \sqrt{15}}{\pi^{2}} \frac{1}{N}<\frac{5}{N} .
$$

To estimate $C_{\text {axial }}(N)$, we still need the actual expression for $\widehat{\varphi}(r, 0)$, which is given in (30). Taking $N=50000$, numerical computation shows that $C_{\text {axial }}(N)=2.90278 \ldots$; hence it follows that

$$
2.90278 \leq C_{\text {axial }} \leq 2.90289 .
$$

Estimating the value of $C_{\text {main }}$. We shall estimate the diagonal terms first. We have

$$
g_{1}(t)=-240 t(1-t)
$$


from which we obtain

$$
\left|\widehat{g}_{1}(0)\right|=40
$$

and

$$
\begin{aligned}
\left|\partial_{1} \partial_{2} F\left(t_{1}, t_{2}\right)\right| & =\left|1200\left(1-t_{1}\right)\left(1-t_{2}\right)\left(2-t_{1}-t_{2}\right)^{4}+280\left(2-t_{1}-t_{2}\right)^{6}-40\right| \\
& \leq 1200\left(1-t_{1}\right)\left(1-t_{2}\right)\left(2-t_{1}-t_{2}\right)^{4}+280\left(2-t_{1}-t_{2}\right)^{6}+40
\end{aligned}
$$

from which we obtain

$$
\left\|\Psi_{\partial_{1} \partial_{2} F}\right\|_{L^{1}\left([0,1)^{2}\right)} \leq 80 .
$$

Hence by (22), we obtain the estimate

$$
|\widehat{\varphi}(r, r)| \leq \frac{30}{\pi^{2}} \frac{1}{r^{2}}
$$

from which it follows that

$$
\sum_{|r|=N+1}^{\infty}|\widehat{\varphi}(r, r)| \leq \frac{60}{\pi^{2}} \frac{1}{N} .
$$

We next estimate $\widehat{\varphi}\left(r_{1}, r_{2}\right)$ in the case when $r_{1} \neq r_{2}$. We begin by noting that

$$
\widehat{g}_{1}\left(r_{1}-r_{2}\right)=\frac{120}{\pi^{2}\left(r_{1}-r_{2}\right)^{2}}, \quad r_{1} \neq r_{2} .
$$

We have

$$
\begin{gathered}
g_{2}(t)=240(1+5 t(1-t)), \quad g_{3}(t)=240\left(-12-15 t+20 t^{2}\right), \\
h_{2}(t)=-40+280(1-t)^{6}, \quad h_{3}(t)=-1680(1-t)^{5}, \\
\partial_{1}^{2} \partial_{2}^{2} F\left(t_{1}, t_{2}\right)=14400\left(2-t_{1}-t_{2}\right)^{2}\left(5+t_{1}^{2}+t_{2}^{2}-5 t_{1}-5 t_{2}+3 t_{1} t_{2}\right),
\end{gathered}
$$

from which we obtain

$$
\begin{gathered}
\widehat{g}_{2}\left(r_{1}-r_{2}\right)=-\frac{600}{\pi^{2}\left(r_{1}-r_{2}\right)^{2}}, \quad r_{1} \neq r_{2}, \\
\left|\widehat{h}_{2}\left(r_{1}\right)\right| \leq \frac{280}{\pi\left|r_{1}\right|}, \quad r_{1} \neq 0, \\
\left|\widehat{g}_{3}\left(r_{1}-r_{2}\right)\right| \leq 3080, \quad\left|\widehat{h}_{3}\left(r_{1}\right)\right| \leq 280, \quad\left\|\Psi_{\partial_{1}^{2} \partial_{2}^{2} F}\right\|_{1}=2800 .
\end{gathered}
$$

Putting these together, we finally obtain the estimate

$$
\left|\widehat{\varphi}\left(r_{1}, r_{2}\right)\right| \leq \frac{105}{\pi^{4}} \frac{1}{\left|r_{1} r_{2}\right|\left(r_{1}-r_{2}\right)^{2}}+\frac{420}{\pi^{4}} \frac{1}{r_{1}^{2} r_{2}^{2}} .
$$

The following is a simple lemma:

Lemma 3. For any $N \geq 1$, one has

$$
\sum_{R=N+1}^{\infty} \sum_{\substack{\max \left(\left|r_{1}\right|,\left|r_{2}\right|\right)=R \\ \min \left(\left|r_{1}\right|,\left|r_{2}\right|\right) \neq 0}} \frac{1}{r_{1}^{2} r_{2}^{2}}<\frac{4 \pi^{2}}{3} \frac{1}{N}
$$


and

$$
\sum_{R=N+1}^{\infty} \sum_{\substack{\max \left(\left|r_{1}\right|,\left|r_{2}\right|\right)=R \\ \min \left(\left|r_{1}\right|,\left|r_{2}\right|\right) \neq 0 \\ r_{1} \neq r_{2}}} \frac{1}{\left|r_{1} r_{2}\right|\left(r_{1}-r_{2}\right)^{2}}<4\left(\frac{\pi^{2}}{3}+1\right) \frac{1}{N}
$$

Proof. The first inequality simply follows from

$$
\sum_{\substack{\max \left(\left|r_{1}\right|,\left|r_{2}\right|\right)=R \\ \min \left(\left|r_{1}\right|,\left|r_{2}\right|\right) \neq 0}} \frac{1}{r_{1}^{2} r_{2}^{2}}=\frac{8}{R^{2}} \sum_{r=1}^{R-1} \frac{1}{r^{2}} \leq \frac{4 \pi^{2}}{3} \frac{1}{R^{2}}
$$

For the second inequality, we first use the symmetries to write

$$
\sum_{\substack{\max \left(\left|r_{1}\right|,\left|r_{2}\right|\right)=R \\ \min \left(\left|r_{1}\right|,\left|r_{2}\right|\right) \neq 0 \\ r_{1} \neq r_{2}}} \frac{1}{\left|r_{1} r_{2}\right|\left(r_{1}-r_{2}\right)^{2}}=\frac{4}{R} \sum_{r=1}^{R-1} \frac{1}{r(R-r)^{2}}+\frac{4}{R} \sum_{r=1}^{R} \frac{1}{r(R+r)^{2}}-\frac{1}{2 R^{4}} .
$$

Using the identity

$$
\frac{1}{r(R-r)^{2}}=\frac{1}{R r(R-r)}+\frac{1}{R(R-r)^{2}}
$$

and the Cauchy-Schwarz inequality we have

$$
\frac{4}{R} \sum_{r=1}^{R-1} \frac{1}{r(R-r)^{2}}=\frac{4}{R^{2}}\left(\sum_{r=1}^{R-1} \frac{1}{r(R-r)}+\sum_{r=1}^{R-1} \frac{1}{(R-r)^{2}}\right)<\frac{4 \pi^{2}}{3} \frac{1}{R^{2}} .
$$

For the remaining terms, we use the trivial estimate

$$
\frac{4}{R} \sum_{r=1}^{R} \frac{1}{r(R+r)^{2}}-\frac{1}{2 R^{4}}<\frac{4}{R^{2}} .
$$

Hence

$$
\sum_{\substack{\max \left(\left|r_{1}\right|,\left|r_{2}\right|\right)=R \\ \min \left(\left|r_{1}\right|,\left|r_{2}\right|\right) \neq 0 \\ r_{1} \neq r_{2}}} \frac{1}{\left|r_{1} r_{2}\right|\left(r_{1}-r_{2}\right)^{2}}<4\left(\frac{\pi^{2}}{3}+1\right) \frac{1}{R^{2}}
$$

and the result follows.

If we define

$$
C_{\text {main }}(N)=\sum_{R=1}^{N} \sum_{\substack{\max \left(\left|r_{1}\right|,\left|r_{2}\right|\right)=R \\ \min \left(\left|r_{1}\right|,\left|r_{2}\right|\right) \neq 0}}\left|\widehat{\varphi}\left(r_{1}, r_{2}\right)\right|
$$

then we have

$$
0 \leq C_{\text {main }}-C_{\text {main }}(N)<\left(\frac{340}{\pi^{2}}+\frac{420}{\pi^{4}}\right) \frac{1}{N}<\frac{40}{N}
$$


For $N=4000$, numerical computation using the formulas (31) and (32) reveals that $C_{\text {main }}(N)=4.75145 \ldots$; hence with the above error estimate, we have

$$
4.75145 \leq C_{\text {main }} \leq 4.76146 .
$$

Explicit expressions for $\widehat{\varphi}\left(r_{1}, r_{2}\right)$. The following formulas have been computed using Mathematica, though it is also possible to compute them easily using the iterative procedure based on integration by parts which was outlined in this section earlier.

(32) $\widehat{\varphi}(r, s)$

$$
\begin{aligned}
\widehat{\varphi}(r, 0)= & \frac{15}{4 \pi^{2} r^{2}}\left(1-\frac{6}{\pi^{2} r^{2}}+\frac{45}{\pi^{4} r^{4}}-\frac{135}{\pi^{6} r^{6}}\right) \\
& -i \frac{60}{7 \pi^{3} r^{3}}\left(1+\frac{63}{8 \pi^{2} r^{2}}-\frac{315}{8 \pi^{4} r^{4}}+\frac{945}{16 \pi^{6} r^{6}}\right), \\
\widehat{\varphi}(r, r)= & \frac{10}{\pi^{2} r^{2}}\left(1-\frac{21}{\pi^{2} r^{2}}+\frac{315}{2 \pi^{4} r^{4}}-\frac{945}{2 \pi^{6} r^{6}}\right) \\
& +i \frac{55}{\pi^{3} r^{3}}\left(1-\frac{126}{11 \pi^{2} r^{2}}+\frac{630}{11 \pi^{4} r^{4}}-\frac{945}{11 \pi^{6} r^{6}}\right),
\end{aligned}
$$

$$
\begin{aligned}
= & -\frac{1575}{4 \pi^{8} r^{6}(r-s)^{2}}+\frac{525}{4 \pi^{6} r^{4}(r-s)^{2}}-\frac{35}{2 \pi^{4} r^{2}(r-s)^{2}}-\frac{1575}{4 \pi^{8}(r-s)^{2} s^{6}} \\
& +\frac{225}{2 \pi^{8} r(r-s)^{2} s^{5}}+\frac{525}{4 \pi^{6}(r-s)^{2} s^{4}}+\frac{225}{2 \pi^{8} r^{2}(r-s)^{2} s^{4}}+\frac{225}{2 \pi^{8} r^{3}(r-s)^{2} s^{3}} \\
& -\frac{75}{2 \pi^{6} r(r-s)^{2} s^{3}}-\frac{35}{2 \pi^{4}(r-s)^{2} s^{2}}+\frac{225}{2 \pi^{8} r^{4}(r-s)^{2} s^{2}}-\frac{75}{2 \pi^{6} r^{2}(r-s)^{2} s^{2}} \\
& +\frac{225}{2 \pi^{8} r^{5}(r-s)^{2} s}-\frac{75}{2 \pi^{6} r^{3}(r-s)^{2} s}+\frac{5}{\pi^{4} r(r-s)^{2} s} \\
& +i\left(-\frac{1575}{4 \pi^{9} r^{7}(r-s)^{2}}+\frac{525}{2 \pi^{7} r^{5}(r-s)^{2}}-\frac{105}{2 \pi^{5} r^{3}(r-s)^{2}}-\frac{1575}{4 \pi^{9}(r-s)^{2} s^{7}}\right. \\
& +\frac{225}{2 \pi^{9} r(r-s)^{2} s^{6}}+\frac{525}{2 \pi^{7}(r-s)^{2} s^{5}}+\frac{225}{2 \pi^{9} r^{2}(r-s)^{2} s^{5}}+\frac{225}{2 \pi^{9} r^{3}(r-s)^{2} s^{4}} \\
& -\frac{75}{\pi^{7} r(r-s)^{2} s^{4}}-\frac{105}{2 \pi^{5}(r-s)^{2} s^{3}}+\frac{225}{2 \pi^{9} r^{4}(r-s)^{2} s^{3}}-\frac{75}{\pi^{7} r^{2}(r-s)^{2} s^{3}} \\
& +\frac{225}{2 \pi^{9} r^{5}(r-s)^{2} s^{2}}-\frac{75}{\pi^{7} r^{3}(r-s)^{2} s^{2}}+\frac{15}{\pi^{5} r(r-s)^{2} s^{2}}+\frac{225}{2 \pi^{9} r^{6}(r-s)^{2} s} \\
& \left.-\frac{75}{\pi^{7} r^{4}(r-s)^{2} s}+\frac{15}{\pi^{5} r^{2}(r-s)^{2} s}\right) .
\end{aligned}
$$




\section{References}

[1] G. Hofmeister, Thin bases of order two, J. Number Theory 86 (2001), 118-132.

[2] W. Klotz, Eine obere Schranke für die Reichweite einer Extremalbasis zweiter Ordnung, J. Reine Angew. Math. 238 (1969), 161-168.

[3] L. Moser, On the representation of $1,2, \ldots, n$ by sums, Acta Arith. 6 (1960), 11-13.

[4] L. Moser, J. R. Pounder and J. Riddell, On the cardinality of h-bases for $n$, J. London Math. Soc. 44 (1969), 397-407.

[5] A. Mrose, Untere Schranken für die Reichweiten von Extremalbasen fester Ordnung, Abh. Math. Sem. Univ. Hamburg 48 (1979), 118-124.

[6] H. Rohrbach, Ein Beitrag zur additiven Zahlentheorie, Math. Z. 42 (1937), 1-30.

Courant Institute

New York University

New York, NY 10012, U.S.A.

E-mail: gunturk@courant.nyu.edu
Department of Mathematics Lehman College (CUNY) Bronx, NY 10468, U.S.A. E-mail: melvyn.nathanson@lehman.cuny.edu

Received on 16.3.2005

and in revised form on 11.1.2006 